\title{
Sehr geehrte Damen und Herren, liebe DGLRM-Mitglieder,
}

die letzten Wochen standen deutlich im Zeichen der Diskussionen um die Zukunft der flugmedizinischen Fortbildung in Deutschland und des Erhalts der Deutschen Akademie für Flugmedizin (DAF).

\section{Zukunft der DAF}

Dazu gab es intensive Gespräche im Vorstand und mit dem Vorstandsrat sowie zwischen der Lufthansa, dem DLR und einem weiteren potenziellen Anbieter Prof. David Groneberg, Direktor des Instituts für Arbeitsmedizin, Sozialmedizin und Umweltmedizin der Johann Wolfgang Goethe-Universität Frankfurt am Main. Während ich diese Zeilen schreibe, sind diese noch nicht abgeschlossen und eine Entscheidung noch nicht getroffen. Festhalten kann ich zu diesem Zeitpunkt jedoch, dass alle Beteiligten an dieser Diskussion daran interessiert sind, die DAF zu erhalten und versuchen, die günstigsten Bedingungen dafür $\mathrm{zu}$ eruieren. Im Vorstand der DGLRM diskutieren wir gerade besonders intensiv über die Rolle unserer eigenen Gesellschaft in der zukünftigen Weiterbildungslandschaft in Deutschland. Dabei spielen auch Überlegungen zu einer schrittweisen Erweiterung unserer Gesellschafteranteile eine Rolle, die das Ziel verfolgen, zukünftig einen größeren Einfluss auf die flugmedizinische Weiterbildung in Deutschland nehmen zu können. Wir wissen alle, dass die Zeit drängt und suchen nach der für alle Beteiligten günstigsten Lösung. Sobald es hier eine endgültige Entscheidung gibt, werde ich Sie auf der Homepage darüber informieren.

\section{DGLRM ist Mitglied der AWMF}

Ich kann Ihnen die erfreulichen Mitteilung machen, dass die DGLRM auf der Delegiertenkonferenz der Arbeitsgemeinschaft der Wissenschaftlichen Medizinischen Fachgesellschaften e.V. (AWMF), die am 7. November dieses Jahres in Frankfurt stattgefunden hat, als Mitglied in diese Arbeitsgemeinschaft aufgenommen wurde. Dies ist insbesondere für unsere AG „Leitlinien, Empfehlungen und Standards in der Flugmedizin “ von großer Bedeutung. Hier können nun in Zusam- menarbeit mit anderen der Wissenschaft verbundenen medizinischen Fachgesellschaften evidenz- und konsensbasierte Leitlinien erarbeitet werden.

\section{DGLRM-Sitzungen bei AsMA-Tagung 2016}

Zur nächsten Jahrestagung der Aerospace Medical Association (AsMA), die vom 24. bis 28. April 2016 in Atlantic City, NY, USA stattfinden wird, haben wir als DGLRM 2 Sitzungen angemeldet.

Eine, die traditionell in deutscher Sprache abgehalten werden wird, soll unter dem Titel: „Clinical case presentations: How to decide according to the guidelines?" laufen. Die zweite wird sich mit der „Diversity in aviation and space medicine" beschäftigen und englischsprachig sein. An dieser Stelle möchte ich ganz besonders Herrn Prof. Dr. Hinkelbein für die Organisation und allen Kollegen, die hierzu beigetragen haben, für ihr Engagement danken.

Tredici Award an Dr. Claudia Stern Auf der diesjährigen Jahrestagung der AsMA in Orlando ist unter den während der Honors Night Ausgezeichneten

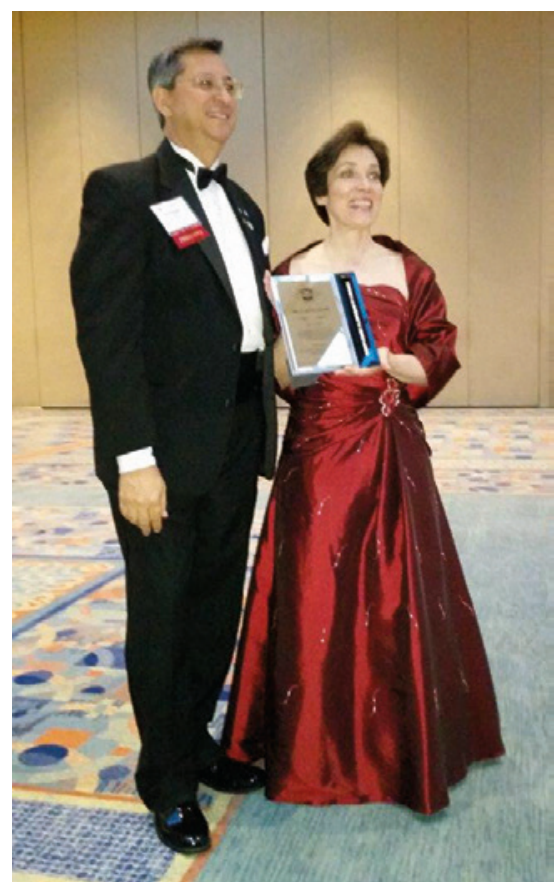

Dr. Claudia Stern erhält vom Präsidenten der AsMA, Dr. Philip Scarpa, den Tredici Award. Quelle: Dr. Roland Vermeiren

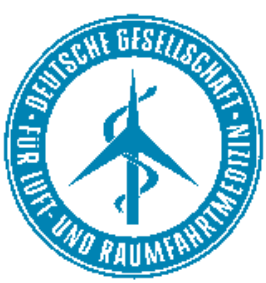

Mitteilungen der

Deutschen Gesellschaft für

Luft- und Raumfahrtmedizin e. V. Nr. 6/2015

DGLRM-Homepage: www.dglrm.de

Geschäftsstelle der DGLRM

Christine Gens

Postfach 870204

13162 Berlin

E-Mail: christine.gens@dglrm.de

Tel.: 0176/5624-2876

Wir gratulieren

- 65 Jahre, Dr. Jörg Binnewies, Rastede, 03.12.1950

- 75 Jahre, Dr. Peter Krupp,

Bad Zwischenahn, 23.11.1940

- 75 Jahre, Dr. Klaus Lefevre, Sittensen, 02.12.1940

\section{Verantwortlich für den Inhalt} der DGLRM-Seiten

Deutsche Gesellschaft für Luft- und Raumfahrtmedizin e. V. (DGLRM) OFA PD Dr. Carla Ledderhos, Präsidentin Zentrum für Luft- und Raumfahrtmedizin der Luftwaffe

Str. der Luftwaffe 322

82242 Fürstenfeldbruck

carlaledderhos@bundeswehr.org

Redaktionsschluss: 21.01.2015

Bankverbindung

Deutsche Bank Hamburg

Konto-Nr.: 388948200

BLZ: 20070000

IBAN: DE 63200700240388948200 BIC: DEUTDEDBHAM

Änderungen für die Mitgliederkartei bitte an: Dipl. Ing. Christine Gammel

Zentrum für Luft- und Raumfahrtmedizin

der Luftwaffe

Str. der Luftwaffe 322

82242 Fürstenfeldbruck

christinegammel@bundeswehr.org 

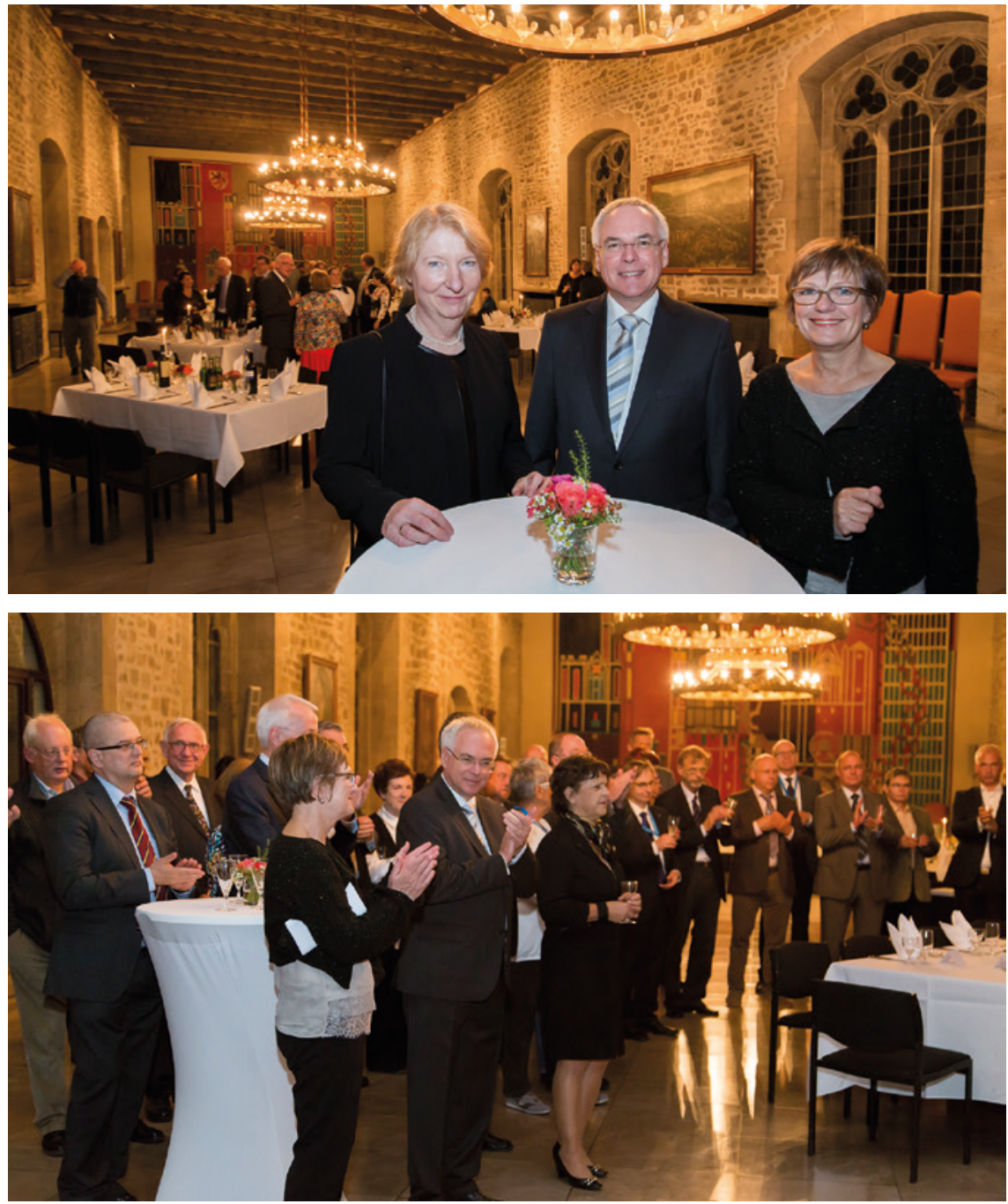

Gesellschaftsabend DGLRM-Tagung in der Dornse im alten Rathaus in Braunschweig.

Oberes Bild v.l.n.r.: Präsidentin der DGLRM mit dem Generalarzt der Luftwaffe Herrn Oberstarzt

Prof. Dr. Schick und der Bürgermeisterin der Stadt Frau Rose-Paul.

Unteres Bild: Teilnehmer des Abends bei der Ansprache.

Quelle: Medienzentrum der Stadt Braunschweig

auch ein Mitglied unserer Gesellschaft, nämlich unsere ehemalige Präsidentin, Frau Dr. Claudia Stern, gewesen. Sie erhielt den „Thomas and Margaret Tredici Award" für ihre Forschungsbeiträge und besondere Expertise und internationale Reputation auf dem Gebiet der Luft- und Raumfahrtopthalmologie.

Wir alle gratulieren ihr sehr herzlich zu diesem ganz besonderen Erfolg!

\section{ECAM 5 in Oslo}

Die 5. European Conference of Aerospace Medicine (ECAM) wird vom 15.-18. September 2016 im Holmenkollen Park Hotel in Oslo stattfinden. 2016 wird die ESAM auf die ersten 10 Jahre ihrer Geschichte zurückblicken können und diese Tagung zum Anlass nehmen, dieses Ereignis würdig zu begehen. Erstmalig wird die ECAM in $\mathrm{Zu}$ sammenarbeit mit der AsMA und natür- lich auch der Norwegischen Gesellschaft für Flugmedizin stattfinden. Der Kongress wird unter dem Motto „Pushing the Limits of Aerospace Medicine together“ stehen und will mit einigen falschen Vorstellungen und Missverständnissen in und über die Flugmedizin aufräumen. Nähere Informationen zum Kongress finden Sie unter www.esam.aero.

\section{Wünsche zum Jahreswechsel}

Liebe Mitglieder der DGLRM, erneut bitte ich Sie, sich auch im nächsten Jahr aktiv und konstruktiv am Leben unserer Gesellschaft zu beteiligen. Gerne nehmen wir Ihre Beiträge für unsere Zeitschrift und/oder die Homepage an. Vielleicht können Sie ja die bevorstehenden freien Tage auch dafür nutzen, sich bereits Gedanken zu Ihrem Beitrag auf unser nächsten, der 54. Jahrestagung, die vom 22.-24. September 2016 im Hubschraubermuseum in Bückeburg stattfinden wird, zu machen. Als Deadline für das Einreichen Ihrer Vortrags- und Posterbeiträge haben wir wieder den 31. März 2016 vorgesehen. Nähere Informationen dazu erhalten Sie im folgenden Heft der FTR und auf unserer Homepage. Als Nachklang von unserer diesjährigen Jahrestagung finden Sie in diesem Heft noch ein paar Schnappschüsse vom Gesellschaftsabend in der „Dornse“, dem Saal im alten Rathaus in Braunschweig.

Für die bevorstehenden Weihnachtsfeiertage wünsche ich Ihnen vor allem besinnliche, frohe Stunden im Kreise Ihrer Familien und dass der Start ins neue Jahr ein Guter wird, wir können nur alle hoffen, dass er auch friedvoll sein wird!

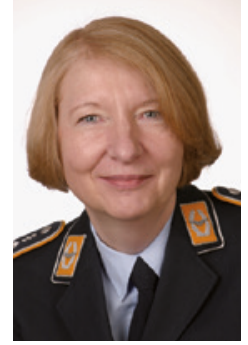

Ihre

Carla Ledderhos
EUSAM - Basic course 26 (englisch)

Termin: 03.09.-11.09.2016

(Ausgebucht, Warteliste) und

EUSAM - Advanced course 26 (englisch)

Termin: 03.12.-11.12.2016
European School of Aviation Medicine

Ort: Medizinischer Dienst der Deutschen Lufthansa auf der Lufthansa-Basis, Flughafen Frankfurt
Kontakt: Sigrid Froese,

Tel: $\quad$ +49(0)69/69691222

Internet: www.flugmed.org/ www.eusam.org 
Zeit: 25.09.2015,

17:00 Uhr bis 18:50 Uhr

Ort: Forschungsflughafen

Braunschweig

Lilienthalplatz 5

38108 Braunschweig

Hermann-Blenk-Saal

Protokoll: Christine Gammel

Teilnehmer: siehe Teilnehmerliste

Anlagen, beim Sekretär (w) anfragbar:

1. Teilnehmerliste

2. Powerpoint Schatzmeister

Tagesordnung

TOP 1 Eröffnung und Begrüßung

TOP 2 Genehmigung der Tagesordnung

TOP 3 Bericht der Präsidentin

TOP 4 Bericht des Sekretärs (w)

TOP 5 Bericht des Schatzmeisters

TOP 6 Bericht der Rechnungsprüfer

TOP 7 Entlastung des Vorstands

TOP 8 Berichte aus den Arbeitsgruppen

TOP 9 Zukunft der flugmedizinischen

Aus- und Weiterbildung in

Deutschland

TOP 10 Zeit und Ort der nächsten Jahrestagung

TOP 11 Sonstiges

TOP 1 Eröffnung und Begrüßung Begrüßung durch Frau Ledderhos

TOP 2 Genehmigung der Tagesordnung

Gewünschte Änderung: Einfügung eines Tagesordnungspunktes „Budget“ nach TOP 5 „Bericht des Schatzmeisters“. Neue Tagesordnung einstimmig angenommen (46 ja, 0 nein, 0 Enthaltungen):

TOP 1 Eröffnung und Begrüßung

TOP 2 Genehmigung der Tagesordnung

TOP 3 Bericht der Präsidentin

TOP 4 Bericht des Sekretärs (w)

TOP 5 Bericht des Schatzmeisters

TOP 6 Budget

TOP 7 Bericht der Rechnungsprüfer

TOP 8 Entlastung des Vorstands

TOP 9 Berichte aus den Arbeitsgruppen

TOP 10 Zukunft der flugmedizinischen Aus- und Weiterbildung in Deutschland
TOP 11 Zeit und Ort der nächsten Jahrestagung

TOP 12 Sonstiges

TOP 3 Bericht der Präsidentin

AsMA

- Positives Feedback zu den beiden deutschen Sessions (deutschsprachig und englischsprachig) auf der AsMA

- 2016 gleiches Vorgehen: eine Session auf Deutsch, eine Session auf Englisch

ECAM

- ECAM 5 in Oslo, Norwegen

- Datum 15. 09.-18.09.2016

- Es wird eine deutsche Session geben

Zukunft der Deutschen Akademie

für Flugmedizin und Reisemedizin (DAF)

- Ende 2015 verlässt Herr Stüben die DAF

- Es ist nicht klar, ob die Lufthansa Gesellschafter der DAF bleibt und wie mit der DAF weiterverfahren wird

- Trotz Abraten der DGLRM wurde über die Lufthansa eine Consultingfirma zur Erstellung eines Gutachtens über die DAF engagiert

- Stellungnahme der DGLRM zur Zukunft der DAF:

- Die DLGRM ist bereit, eine Taskforce zur Zukunft der DAF zu bilden

- Die DGLRM will an der zivilen Ausbildung von Flugmedizinern festhalten

- Vorstellbar wäre eine Verlagerung der flugmedizinischen Ausbildung nach Köln im Rahmen einer Kooperation zwischen dem ZentrLuRMedLw und dem DLR

- Die DGLRM ist außerdem daran interessiert, dass die Lufthansa weiterhin als Gesellschafter aktiv bleibt

Arbeitsgruppen

- AG Geschäftsordnung

- Ein Entwurf für die neue Geschäftsordnung wurde erarbeitet

- Der Entwurf wird auf nächster Vorstandsitzung besprochen

- Nach der Einpflegung von endgültigen Änderungen soll die Geschäftsordnung beschlossen werden

- AG Empfehlungen/Leitlinien

- Identifikation erster Schwerpunkte: Reanimation im Luftfahrzeug, Ausstattung bei MedEvac, Allergien und Fliegen, Luftkrankheit
- Interessenten sind jederzeit gesucht und in der AG herzlich willkommen

- Zunächst Abgabe von Empfehlungen

- Langfristiges Ziel ist aber die Ausarbeitung zu Leitlinien

- Hierzu wurde bereits ein Antrag zur Aufnahme der DGLRM in die Arbeitsgemeinschaft der Wissenschaftlichen Medizinischen Fachgesellschaften (AWMF) gestellt

- Der Bescheid über den Antrag ist im Laufe des Novembers 2015 zu erwarten

Taskforce des Bundesministers für Verkehr und digitale Infrastruktur

- Die Taskforce wurde abgegeben an den Bundesverband der Deutschen Luftverkehrswirtschaft (BDL)

- Trotz erheblicher Bemühung wurde kein Vertreter der DGLRM in die Taskforce berufen

Gestaltung der Jahrestagungen der $\mathrm{Zu}-$ kunft

- Die Jahrestagung der DGLRM soll alle 2 Jahre fest in Köln stattfinden

- Der Tagungsort der jeweils anderen Jahre ist frei wählbar

- Geplant ist eine Zusammenlegung mit dem Tag der Luft und Raumfahrt

- Der Tag der Luft-und Raumfahrt findet alle 2 Jahre jeweils am dritten Sonntag im September statt

- Beginn dieser Routine wäre 2017

- Empfehlung des Vorstandsrats: Der Vorstandsrat möchte die Gestaltung der Jahrestagung nicht bindend festlegen, um zukünftigen Vorständen Handlungsfreiheit zu gewähren, findet den Vorschlag grundsätzlich aber gut.

TOP 4 Bericht des Sekretärs (w)

- Mitgliederzahlen bis zur Jahrestagung 2014: 428

- Austritte 2015: 17

- $\quad$ Eintritte 2015: 23

- Mitgliederzahlen bis zur Jahrestagung 2015: 434

TOP 5 Bericht des Schatzmeisters Siehe Anlage 2

TOP 6 Budget

- Mit den momentanen Mitgliedsbeiträgen können die notwendigen Kosten gerade so gedeckt werden 
- Ergriffene Sparmaßnahmen:

- Abschaffung der Kongressgesellschaft für die Jahrestagung

- Reduktion der Anzahl der Vorstandssitzungen zur Einsparung von Reisekosten

- Kürzung des Budgets der Geschäftsstelle von 400 Euro auf 300 Euro im Monat

- Schatzmeister katalogisiert Einund Ausgaben in Eigenregie, um die Kosten für die Steuerberaterin zu senken

- Gründe für eine Erhöhung des Mitgliedsbeitrags:

- Kosten für die Zeitschrift FTR werden steigen

- Überführung der Homepage auf neue Software wird dringend benötigt, da die momentane Software nicht mehr unterstützt wird

- Es wird dringend neue Tagungstechnik benötigt

- Keinerlei monetäre Förderung von AGs oder Nachwuchswissenschaftlern möglich

- Sponsoren sind für eine kleine Gesellschaft nur schwer zu akquirieren

- Empfehlung des Vorstandsrats: moderate Erhöhung auf 120 Euro

- Meinungsbild aus dem Auditorium:

。 Erhöhung auf 120 Euro/Jahr

$\rightarrow$ Mehrheit

。 Erhöhung 140 Euro/Jahr $\rightarrow$ keiner

。 Beibehaltung 100 Euro/Jahr $\rightarrow$ keiner

- Endgültige Abstimmung über die Neugestaltung des Mitgliedsbeitrags auf der Jahrestagung 2016

TOP 7 Bericht der Rechnungsprüfer

- Herr Kimmich berichtet für die Rechnungsprüfer

- Die Ein- und Ausgaben sind alle korrekt und gut nachvollziehbar aufgeführt

- Es gibt keine Beanstandungen

TOP 8 Entlastung des Vorstands

- Antrag auf Entlastung des Vorstands

- Vorstand entlastet (39 ja, 0 nein, 7 Enthaltungen)

TOP 9 Berichte aus den Arbeitsgruppen

AG Arbeitsmedizin

- Herr Hedtmann berichtet über die Arbeit der AG
- Schwerpunktthemen der AG sind: Kabinenluft, Entlastung von Flugbegleitern im Umgang mit Personen mit eingeschränkter Mobilität, Temperatur im Cockpit

AG Fliegerärztliche Aus- und Weiterbil-

dung

- Herr Pongratz berichtet über die Arbeit der AG

- Zurzeit werden die neuen Entwürfe für die Aus- und Weiterbildungsordnung erarbeitet

- Vor Frühjahr 2016 ist nicht mit den Entwürfen zu rechnen

AG Geschichte der Luft- und Raumfahrtmedizin

- Herr Harsch berichtet über die Arbeit $\operatorname{der} A G$

AG Human Factors

- Herr Glaser berichtet über die Arbeit der AG

AG Leitlinien und Empfehlungen

- Herr Hinkelbein berichtet über die Arbeit der AG

- Publikation: Reanimation im Weltall

- Erstellung Leitlinie Reanimation an Bord von Luftfahrzeugen

- In Zukunft geplant: Reanimation in Mikrogravitation

AG Notfallmedizin und Luftrettung

- Herr Hinkelbein berichtet über die Arbeit der AG

- Aktueller Schwerpunkt: Notfallequipment an Bord von Luftfahrzeugen

AG Militärische Flugmedizin

- Herr Pippig berichtet über die Arbeit der AG

AG Raumfahrtmedizin

- Herr Binnewies berichtet über die Arbeit der AG

TOP 10 Zukunft der flugmedizinischen Aus- und Weiterbildung in Deutschland

Siehe TOP 9 „Berichte aus den Arbeitsgruppen“

TOP 11 Zeit und Ort der nächsten Jahrestagung

Vorbehaltlich anderer Planung:

Bückeburg,

Hubschraubermuseum,

22.09.-24.09.2016

TOP 12 Sonstiges

Zur nächsten Mitgliederversammlung wird eine Aufstellung über die Altersstruktur der Mitglieder der DGLRM gewünscht 